Network Working Group

Request for Comments: 360

C. Holland

UCSD-CC

Category: Protocols, RJE

June 1972

NIC : 10602

\title{
PROPOSED REMOTE JOB ENTRY PROTOCOL
}

Remote job entry is the mechanism whereby a user at one location causes a batch-processing job to be run at some other location. This protocol specifies the Network standard procedures for such a user to communicate over the Network with a remote batch-processing server, causing that server to retrieve a job-input file, process the job, and deliver the job's output file(s) to a remote location. The protocol uses TELNET (to a special standardized logger, not socket 1) connection for all control communication between the user and the server RJE process. The server-site then uses the File Transfer Protocol to retrieve the job-input file and to deliver the output file $(\mathrm{s})$.

There are two types of users: direct users (persons) and user processes. The direct user communicates from an interactive terminal attached to a TIP or any host. This user may cause the input and/or output to be retrieved/sent on a specific socket at the specified host (such as for card readers or printers on a TIP), or the user may have the files transferred by pathname using File Transfer Protocol. The other type of user is an RJE User-process in one remote host communicating with the RJE Server-process in another host. This type of user ultimately receives its instructions from a human user, but through some unspecified indirect means. The command and response streams of this protocol are designed to be readily used and interpreted by both the human user and the user process.

A particular user location may choose to establish the TELNET control connection for each logical job or may leave the control connection open for extended periods. If the control connection is left open, then multiple job-files may be directed to be retrieved or optionally (to servers that are able to determine the end of one logical job by the input stream and form several jobs out of one input file) one continuous retrieval may be done (as from a TIP card reader). This then forms a "hot" card reader to a particular server with the TELNET connection serving as a "job monitor". Since the output is always transferred job at a time per connection to the output socket, the output from this "hot" reader would appear when ready as if to a "hot" printer. Another possibility for more complex hosts is to attach an RJE User-process to a card reader and take instructions from a lead control card, causing an RJE control TELNET to be opened to the appropriate host with appropriate logon and input retrieval 
commands. This card reader would appear to the human user as a Network "host" card reader. The details of this RJE User-process are beyond the scope of this protocol.

\section{GENERAL SPECIFICATIONS}

1. User - A human user at a real terminal or a process that supplies the command control stream causing a job to be submitted remotely will be termed the User. The procedure by which a process user receives its instructions is beyond the scope of this protocol.

2. User TELNET - The User communicates its commands over the Network in Network Virtual Terminal code through a User TELNET process in the User's Host. This User TELNET process initiates its activity via ICP to the standard "RJE logger" socket (socket 5) at the desired RJE-server Host.

3. RJE-server TELNET - The RJE-server process receives its command stream from and sends its response stream to the TELNET channel through an RJE-server TELNET process in the server host. This process must listen for the ICP on the "RJE logger" socket (and cause appropriate ICP socket shifting).

4. TELNET Connection - The command and response streams for the RJE mechanism are via a TELNET-like connection to a special socket with full specifications according to the current NWG TELNET protocol.

5. RJE-Server - The RJE-Server process resides in the Host which is providing Remote Batch Job Entry service. This process receives input from the RJE-Server TELNET, controls access through the "logon" procedure, retrieves input job files, queues jobs for execution by the batch system, responds to status inquiries, and transmits job output files when available.

6. User FTP - All input and output files are transferred under control of the RJE-server process at its initiative. Those files may be directly transferred via Request-for-connection to a specific Host/socket or they may be transferred via File Transfer Protocol. If the later method is used, then the RJEserver acts through its local User FTP process to cause the transfer. This process initiates activity by an active Request-for-connection to the "FTP Logger" in the foreign host. 
7. Server FTP - This process in a remote host (remote from the RJE-server) listens for an ICP from the User FTP and then acts upon the commands from the User FTP causing the appropriate file transfer.

8. FTP - When File Transfer Protocol is used for RJE files, the standard FTP mechanism is used as fully specified by the current NWG FTProtocol.

9. RJE Command Language - The RJE system is controlled by a command stream from the User over the TELNET connection specifying the user's identity (logon), the source of the job input file, the status, altering job status or output disposition. Additional commands affecting output disposition are includable in the job input file. This command language is explicitly specified in a following section of this protocol.

10. RJE Command Replies - Every command input from the User via TELNET and certain other conditions calls for a response message from the RJE-server to the User over the TELNET connection. These messages are formatted in a standardized manner to facilitate interpretation by both human Users and User processes. A following section of this protocol specifies the response messages.

\section{RJE COMMANDS OVER TELNET CONNECTION}

\section{GENERAL CONVENTIONS}

1. All commands will be contained in one input line terminated by the standard TELNET "crlf". The line may be of any length desired by the user (explicitly, not restricted to a physical terminal line width). The characters "cr" and "lf" will be ignored by the RJE-server except in the explicit order "crlf" and may be used as needed for local terminal control.

2. All commands will begin with a recognized command name and may then contain recognized syntactic element strings and free-form variable strings (for userid, pathnames, etc.). Recognized words consist of alphanumeric strings (letters and digits) or punctuation. Recognized alphanumeric string elements must be separated from each other and from unrecognizable strings by at least one blank or a syntactically permitted punctuation. Other blanks may be used freely as desired before or after any syntactic element. The "=" after the command name in all commands except OUT and CHANGE are optional. 
3. Recognized alphanumeric strings may contain upper case letters or lower case letters in any mixture without syntactic differentiation. Unrecognizable strings will be used exactly as presented with full differentiation of upper and lower case input, unless the host finally using the string defines otherwise.

4. There are two types of Unrecognizable strings: final and imbedded. Final strings appear as the last syntactic element of a command and are parsed as beginning with the next non-blank character of the input stream and continuing to the last nonblank character before the "crlf".

Imbedded strings include "job-id" and "job-file-id" in the OUT, CHANGE, and ALTER commands. At present these fields will be left undelimitted since they must only be recognizable by the server host which hopefully can recognize its own job-ids and file-names.

SYNTAX: The following command descriptions are given in a BNF-like syntax. Parenthesized names are non-terminal syntactic elements which are expanded in succeeding syntactic equations. Each equation has the defined name on the left of the ::= and a set of alternative definitions, separated by slashes "/", on the right. The equations for (host-file) and (disp) use the characters "/" " ( )" explicitly in their definitions. In these cases the quotes are not part of the definition, but surround literal text which is part.

USER

$\mathrm{USER}=($ user-id)

This command must be the first command over a new TELNET connection. As such, it initiates a "logon" sequence. The response to this command is either

a) User code in error.

b) Enter password (if usercode ok)

c) Log-on ok, proceed. (if no password required)

Another USER command may be sent by the User at any time to change Users. Further input will then be charged to the new user. A server may refuse to honor a new user command if it is not able to process it in its current stat (during input file transfer, for example), but the protocol permits the USER command at any time 
without altering previous activity. An incorrect subsequent USER command or its following PASS command are to be ignored with error response, leaving the original User logged-in.

It is permissable for a server to close the TELNET connection if the initial USER/PASS commands are not completed within a server specified time period.

It is not required or implied that the "logged-on" User be the user-id used for file transfer or job execution, but only identifies the submitter of the command stream. Servers will establish their own rules relating User-id with the job-executionuser for Job or Output alteration commands.

Successful "log-on" always clears any previous Input or Output default parameters (INID, etc.).

PASS

PASS $=($ password $)$

This command immediately follows a USER command and completes the "log-on" procedure. Although a particular Server may not require a password and has already indicated "log-on ok" after the USER command, every Server must permit a PASS command (and possibly ignore it) and acknowledge it with a "log-on ok" if the log-on is completed.

BYE

BYE

This command terminates a USER and if input transfer is not in progress, closes the TELNET connection. If input is in progress, the connection will remain open for result response and will then close. During the interim, a new USER command (and no other command) is acceptable.

An unexpected close on the TELNET connection will cause the server to take the effective action of an ABORT and BYE.

INID/INPASS

INID $=$ (user-id)

INPASS $=$ (password) 
The specified Userid and Password will be sent in the File Transfer request to retrieve the input file. These parameters are not used by the Server in any other way. If this command does not appear, then the USER/PASS parameters are used.

INPATH / INPUT

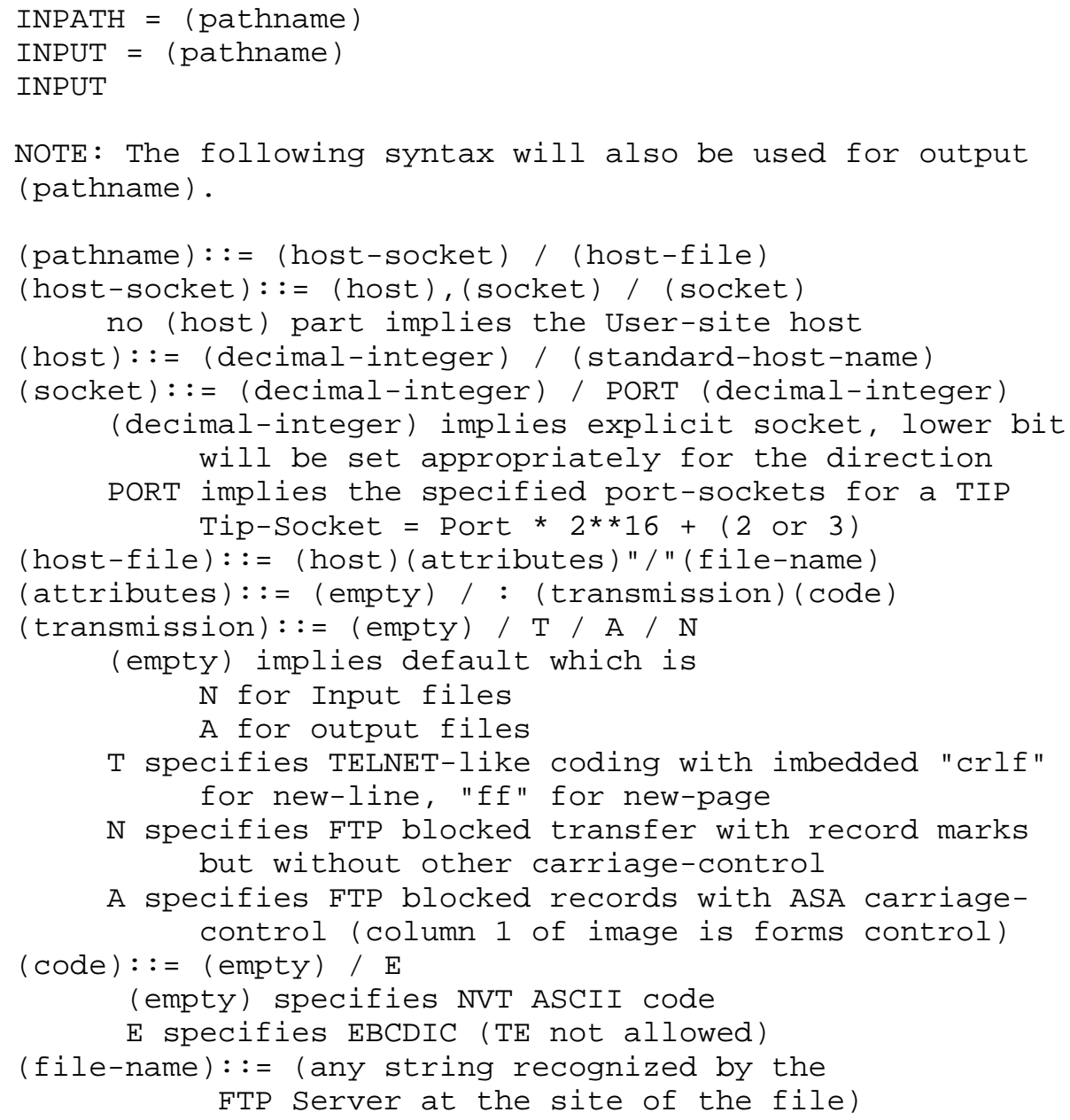

The (pathname) syntax is the general RJE mechanism for specifying a particular file source or destination for input or output. If the (host-socket) form is used then direct transfer will be made by the RJE-Server to the named socket using TELNET-like ASCII. If the (host-file) form is used then the RJE-server will call upon its local FTP-user process to do the actual transfer. The data stream in this mode is either TELNET-like ASCII or blocked records (which may use 
column 1 for ASA carriage-control). Although A mode is permitted on input (column 1 is deleted) the usual mode would be the default $\mathrm{N}$. The output default A would supply carriage-control in the first character of each record ("blank"= single-space, "1"=new-page, etc.), while the optional $\mathrm{N}$ mode would transfer the data only (as to a card punch, etc.).

The (file-name) is an arbitrary Unrecognized string which is saved by RJE-server and sent back over FTP to the FTP-server to retrieve or store the appropriate files.

INPATH or INPUT commands first store the specified (pathname) if one is supplied, and then the INPUT command initiates input. The INPATH name may be used to specify a pathname for later input and the INPUT command without pathname will cause input to initiate over a previously specified pathname. An INPUT "crlf" command with no previous (pathname) specified is illegal.

ABORT

ABORT

This command aborts any input retrieval in progress, discards already received records, and closes the retrieval connection.

Note: ABORT with parameters is an Output Transmission control. OUTUSER/OUTPASS

OUTUSER $=($ user-id) OUTPASS $=$ (password)

The specified Userid and Password will be sent in the File Transfer request to send the output file(s). These parameters are not used by the Server in any other way. If this command does not appear, then the USER/PASS parameters are used. 
OUT

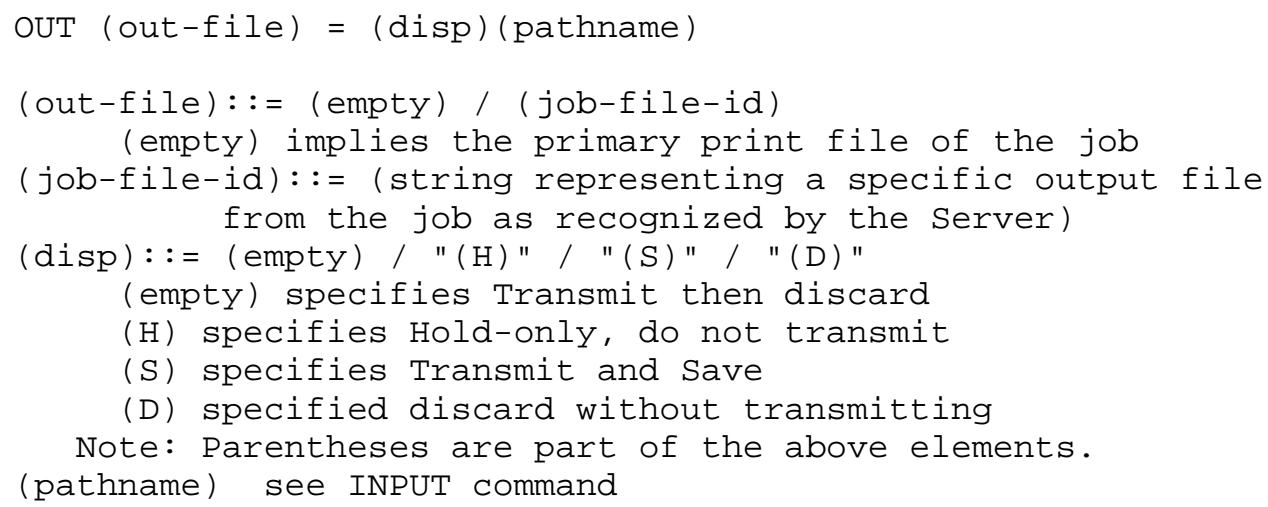

This command specifies the disposition of output file(s) produced by the job. Unspecified files will be Hold-only by default. The OUTUSER, OUTPASS, and OUT commands must be specified before INPUT command to be effective. These commands will affect any following jobs submitted by this USER over this RJE-TELNET connection. A particular job may override these commands by NET control cards on the front of the input file.

Once output disposition is specified by this OUT command or by a NET OUT card, the information is kept with the job until final output disposition, and is modifiable by the CHANGE command.

OUTPUT RE-ROUTE

CHANGE (job-id) (out-file) = (disp) (pathname)

This command changes the output disposition supplied with the job submission. The (job-id) is assumed recognizable by the RJEserver, who may verify if this USER is authorized to modify the specified job. After the job is identified, the other information has the same syntax and semantics as the original OUT command. CHANGE command may be specified for a job-file-id which was not mentioned at submission time and has the same effect as an original OUT command. 
OUTPUT CONTROLS DURING TRANSMISSION

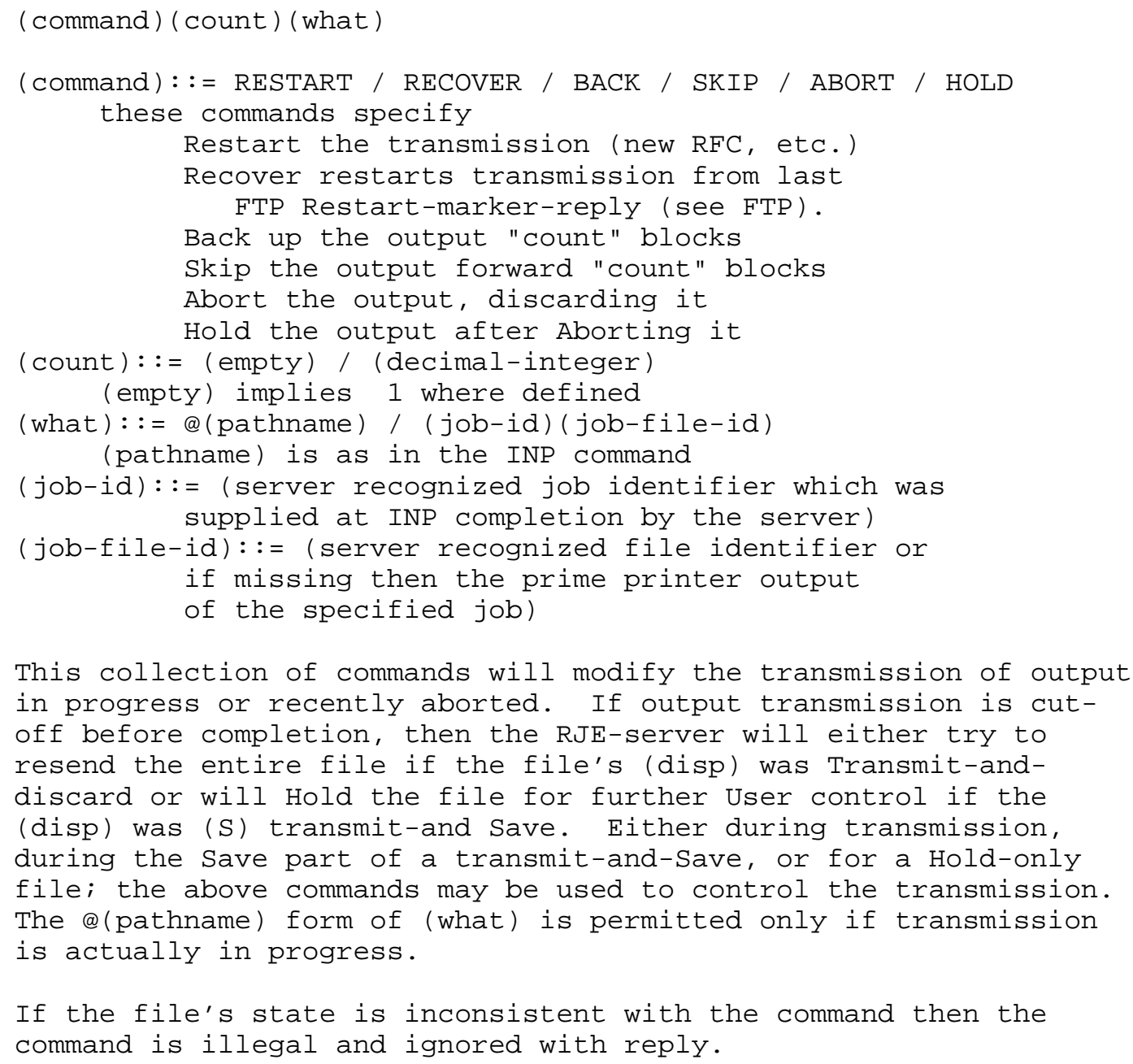




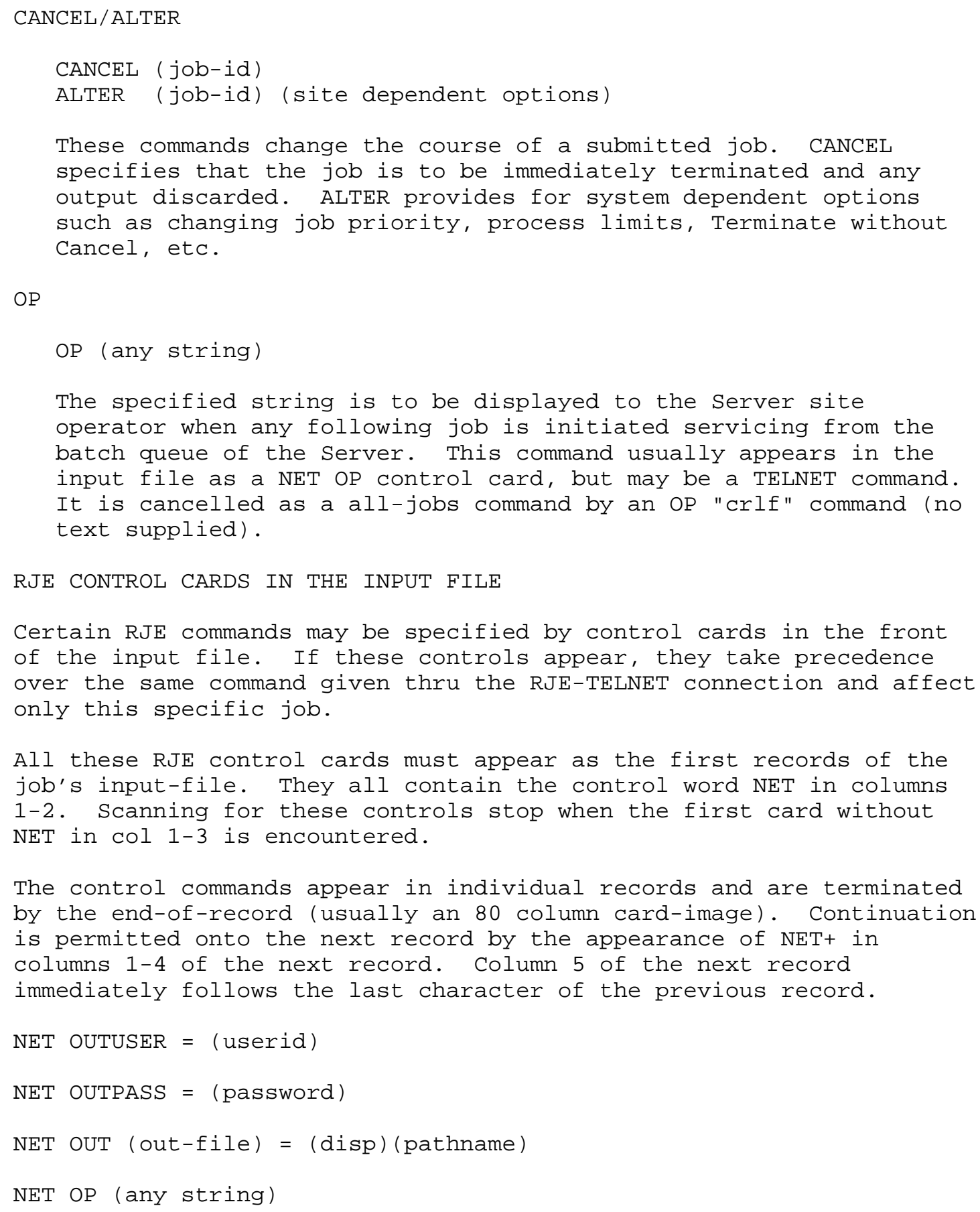


See the corresponding TELNET commands for details. One option permitted by the NET OUTUSER and NET OUT controls not possible from the TELNET connection is specification of different OUTUSERs for different OUTs, since the TELNET stores and supplies only an initial OUTUSER, but the controls may change OUTUSERs before each OUT control is encountered.

RJE USE OF FILE TRANSFER PROTOCOL

Most non-TIP files will be transferred to or from the RJE-server through the FTP process. RJE-server will call upon its local FTPuser supplying the Host, File-pathname, User-id, Password, and Mode of the desired transfer. FTP-user will then connect to its FTPserver counterpart in the specified host and set up a transfer path. Data will then flow through the RJE-FTP interface in the Server, over the Network, from/to the foreign FTP-server and then from/to the specified File-pathname in the foreign host's file storage space. On output files, the file-pathname may be recognized by the foreign host as directions to a printer or the file may simply be stored; a UserRJE-process can supply output (pathname) by default which is recognized by its own server-FTP as routing to a printer.

Although many specifics of the RJE-Server/User-FTP interface are going to be site dependent, there are several FTP options which will be used in a standard way by RJE-Servers:

1. A new FTP connection will be initiated for each file to be transferred. The connection will be opened with the RJE User supplied User-id (OUTUSER or INUSER) and Password.

2. The data bytesize will be 8 bits.

3. The FTP Type, Structure, and Mode parameters are determined by the RJE transfer direction (I/O), the (transmission and (code) options supplied by the User: 


\begin{tabular}{|c|c|c|c|c|c|}
\hline $\mathrm{I} / \mathrm{O}$ & (TRANS) & $(\mathrm{CODE})$ & FTP TYPE & RUCTURE & MODE \\
\hline$I *$ & $\mathrm{~N}$ & - & Ascii & $\mathrm{R}$ & Hasp \\
\hline I & $\mathrm{N}$ & $\mathrm{E}$ & Image & $\mathrm{R}$ & Hasp \\
\hline I & $\mathrm{T}$ & - & Ascii & $\mathrm{F}$ & Ascii \\
\hline I & A & - & Ascii & $\mathrm{R}$ & Hasp \\
\hline I & A & $E$ & Image & $\mathrm{R}$ & Hasp \\
\hline $0 *$ & A & - & Ascii-print & $\mathrm{R}$ & Hasp \\
\hline $\mathrm{O}$ & A & $E$ & Ebcdic-print & $\mathrm{R}$ & Hasp \\
\hline 0 & $\mathrm{~N}$ & - & Ascii & $\mathrm{R}$ & Hasp \\
\hline O & $\mathrm{N}$ & $E$ & Image & $\mathrm{R}$ & Hasp \\
\hline 0 & $\mathrm{~T}$ & - & Ascii-print & $\mathrm{F}$ & Ascii \\
\hline
\end{tabular}

Note: The I* and $\mathrm{O}^{*}$ are the default cases.

4. The service commands used will be Retrieve for input and Append (with create) for output. The FTP pathname will be the (filename) supplied by the RJE User.

5. On output in Hasp form, the User-FTP at the RJE-Server site will send Restart-markers at periodic intervals (like every 100 lines, or sol, and will remember the latest Restart-marker-reply with the file. If the file transfer is not completed and the (disp) is (S) then the file will be held pending User intervention. The User may then use the RECOVER command to cause a FTP restart at the last remembered Restart-marker-reply.

6. The FTP Abort command will be used for the RJE ABORT and CANCEL commands.

The specific form of the FTP commands used by an RJE-Server site, and the order in which they are used will not be specified in this protocol.

Errors encountered by FTP fall into three categories: a) access errors or no storage space error; b) command format errors; and c)transfer failure errors. Since the commands are created by the RJE-Server process, an error is a programming problem and should be logged for attention and the situation handled as safely as possible.

Transmission failure or access failure on input cause an effective ABORT and user notification. Transmission failure on output causes RESTART or Save depending on (disp). (see OUT command.) Access failure on output is a problem since the User may not be accessible. A status response should be queued for that user, should he happen to inquire; a $(\mathrm{disp})=(\mathrm{S})$ file should be Held; and a (disp) $=($ empty) transmit-and-discard file should be temporarily held and then discarded after a reasonable time if not claimed. 
REPLIES OVER THE TELNET CONNECTION

Each action of the RJE-server, including entry of each TELNET command, is noted over the TELNET connection to the User. These RJE-server replies are formatted for Human or Process interpretation. They consist of a leading 3-digit numeric code followed by a blank followed by a text explanation of the message. The numeric codes are assigned by groups for future expansion to hopefully cover other protocols besides RJE (like FTP). The numeric code is designed for ease of interpretation by processes. The three digits of the code are interpreted as follows:

a) The first digit specifies the "type" of response indicated:

000 These "replies" are purely informative, and are issued voluntarily by the Server to inform a User of some state of the server's system.

100 Replies to a specific status inquiry. These replies server as both information and as acknowledgement of the status request.

200 Positive acknowledgement of some previous command/request. The reply 200 is a generalized "ok" for commands which require no other comment. Other $2 \mathrm{xx}$ replies are specified for specific successful actions.

300 Incomplete information supplied so far. No major problem, but activity can not proceed with the input supplied.

400 Unsuccessful reply. A request was correctly specified, but could not be correctly completed. Further attempts will require User commands.

500 Incorrect or illegal command. The command or its parameters were invalid or incomplete from a syntactic view, or the command is inconsistent with a previous command. The command in question has been totally ignored.

600-900 Reserved for expansion.

b) The second digit specifies the general subject to which the response refers:

x00-x29 General purpose replies, not assignable to other subjects.

x30 Primary access. There replies refer to the attempt to "logon" to a server service (RJE, FTP, etc.). 
x40 Secondary access. The primary server is commenting on its ability to access a secondary service (RJE must log-on to a remote FTP service).

x50 FTP results.

x60 RJE results.

x70-x99 Reserved for expansion.

c) The final digit specifies a particular message type. Since the code is designed for an automation process to interpret, it is not necessary for every variation of a reply to have a unique number, only that the basic meaning have a unique number. The text of a reply can explain the specific reason for the reply to a human User.

Each TELNET line (ended by "crlf") from the Server is intended to be a complete reply message. If it is necessary to continue the text of a reply onto following lines, then those continuation replies contain the special reply code of three blanks.

The assigned reply codes relating to RJE are:

000 General information message (time of day, etc.)

030 Server availability information

050 FTP commentary or user information

060 RJE or Batch system commentary or information

100 System status reply

150 File status reply

151 Directory listing reply

160 RJE system general status reply

161 RJE job status reply

200 Last command received ok

201 An ABORT has terminated activity, as requested

202 ABORT request ignored, no activity in progress

203 The requested Transmission Control has taken effect

230 LOG-on completed

231 Log-off completed, goodbye.

232 Log-off noted, will complete when transfer done

240 File transfer has started

250 FTP file transfer started ok

251 FTP Restart-marker-reply

Text is: MARK YYyy = mmmm

where yyyy is data stream marker value (yours)

and mmm is receiver's equivalent mark (mine)

252 FTP transfer completed ok

253 Rename completed 


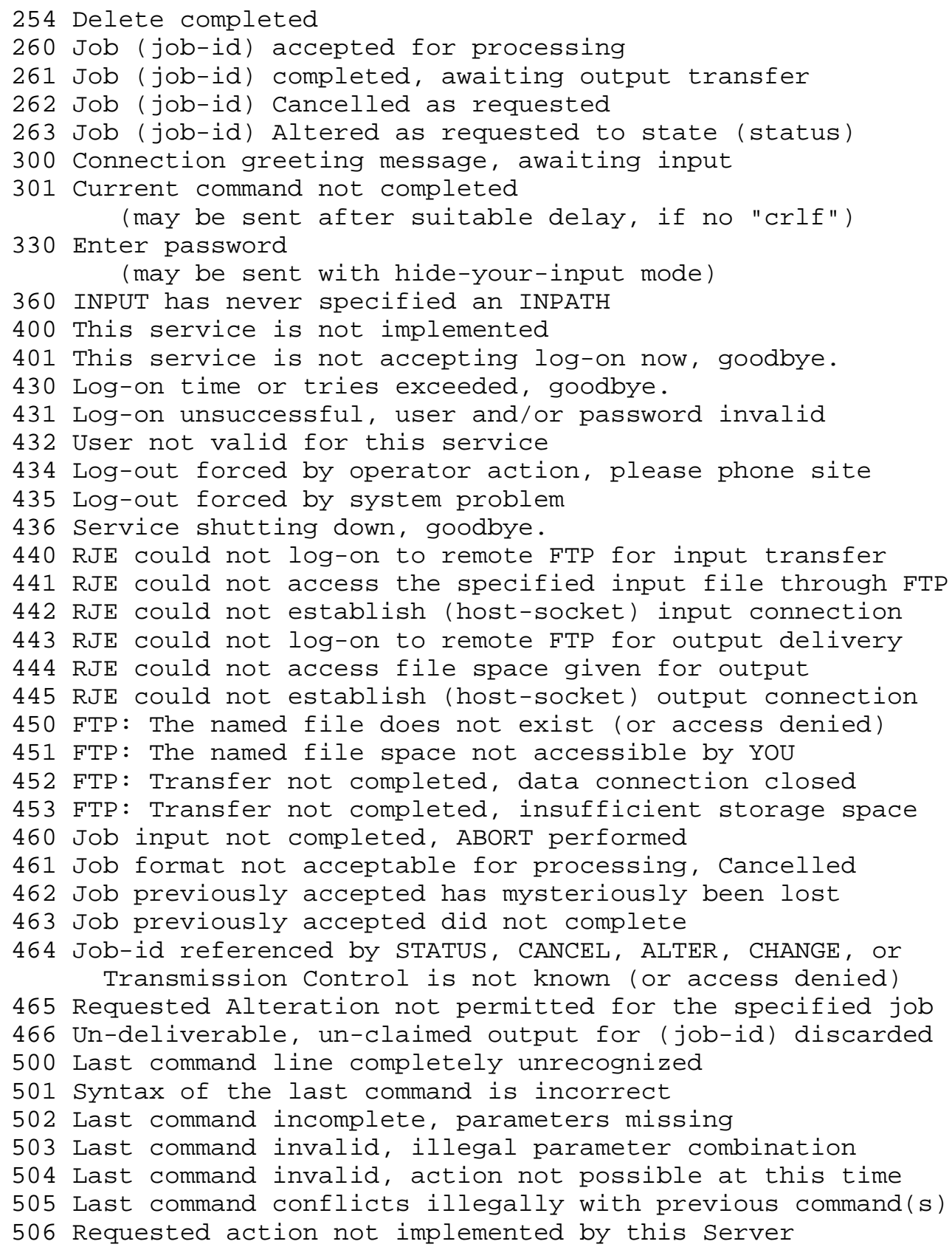


SEQUENCING OF COMMANDS AND REPLIES

The communication between the User and Server is intended to be an alternating dialogue. As such, the User issues an RJE command and the Server responds with a prompt primary reply. The User should wait for this initial success or failure response before sending further commands.

A second type of reply is sent by Server asynchronously with respect to User commands. These replies report on the progress of a job submission caused by the INPUT command and as such are secondary replies to that command

The final class of Server "replies" are strictly informational and may arrive at any time. These "replies" are listed below as spontaneous.

COMMAND-REPLY CORRESPONDENCE TABLE

\begin{tabular}{|c|c|c|}
\hline COMMAND & Success & Fail \\
\hline------- & ------- & ---- \\
\hline USER & 230,330 & $430,431,432,500-505$ \\
\hline PASS & 230 & $430,431,432,500-505$ \\
\hline BYE & 231,232 & $500-505$ \\
\hline INID & 200 & $500-505$ \\
\hline INPASS & 200 & $500-505$ \\
\hline INPATH & 200 & $500-505$ \\
\hline INPUT & 240 & $360,440-442,500-505$ \\
\hline sec. Input retrieval & 260 & 460,461 \\
\hline sec. Job execution & 261 & 462,463 \\
\hline sec. Output Transmissic & $o n--$ & $443,444,445,446$ \\
\hline ABORT (input) & 201,202 & $500-505$ \\
\hline OUTUSER & 200 & $500-505$ \\
\hline OUTPASS & 200 & $500-505$ \\
\hline OUT & 200 & $500-505$ \\
\hline CHANGE & 200 & $500-505$ \\
\hline \multicolumn{3}{|l|}{ RESTART/RECOVER/BACK/ } \\
\hline SKIP / ABORT (output) / HOLD & 203 & $464,500-506$ \\
\hline STATUS & $1 x x$ & $464,500-505$ \\
\hline CANCEL & 262 & $464,500-506$ \\
\hline ALTER & 263 & $464,465,500-506$ \\
\hline OP & 200 & $500-505$ \\
\hline Spontaneous & $0 x x, 300,301$ & $434-436$ \\
\hline \multicolumn{3}{|c|}{$\begin{array}{l}\text { Note: For commands appearing on cards, the } 200 \text { is not sent } \\
\text { but the 500-505 errors may be "asynchronously" sent. }\end{array}$} \\
\hline
\end{tabular}


TYPICAL RJE SCENARIOS

1. TIP USER WANTING HOT CARD READER TO HOSTX

a) TIP user opens TELNET connection to HOSTX socket 5

b) Commands sent over TELNET to RJE

USER=myself

PASS $=$ dorws sap

OUT=PORT 7

INPUT=PORT 5

c) RJE-server connects to the User's host port 5 and begins reading. When end-of-job card is recognized, the job is queued to run. The connection to the card reader is still open for more input as another job

d) The first job finishes. A connection to the Users host port 7 is established by RJE-server and the output is sent as an NVT stream.

e) Continue at any time with another deck at step c) .

2. TIP WITH JOB-AT-A-TIME CARD READER

a) thru d) the same but User closes Reader after the deck

e) The output finishes and the printer connection closes.

f) INPUT may be typed any time after step c) finishes and another job will be entered starting at $c$ ).

3. HOSTA USER RUNS JOB AT HOSTC, INPUT FROM HOSTB

a) User TELNET connects to HOSTC socket 5 for RJE

USER=roundabout

PASS $=$ a a abbbc

OUTUSER=roundab1

OUT $=:$ E/.sysprinter

OUT puncher $=(\mathrm{s}) \mathrm{HOSTB}: \mathrm{NE} / \mathrm{my} \cdot$ savepunch

INUSER=rounder

INPASS $=\mathrm{x} \cdot \mathrm{x} \cdot \mathrm{x}$

INPUT $=$ HOSTB $: E / m y \cdot j o b i n p u t$

b) The RJE-server has FTP retrieve the input from HOSTB using Userid of "rounder" and Password of "x.x.x" for file named "my · jobinput" .

c) The job finishes. RJE-server uses FTP to send two files: the print output is sent to HOSTA in EBCDIC with ASA carriage control to file ".sysprinter" while the file known as "puncher" is sent to HOSTB in EBCDIC without carriage-control to file "my. savepunch".

d) when the outputs finish, RJE-server at HOSTC discards the print file but retains the "puncher" file.

e) The User who had signed out after job submission has gotten his output and checked his file "my.savepunch" at HOSTB. He deletes the saved copy at HOSTC by re-calling RJE at HOSTC. USER=roundabout 
PASS $=$ a aabbbcc

ABORT job123 puncher

or by

CHANGE job123 puncher $=$ (D) 